\title{
Análisis de la influencia de la orientación profesional en los jóvenes en situación de abandono escolar
}

\section{Analysis of the influence of career guidance on early school leaving}

\author{
Ana Isabel Holgueras González ${ }^{1}$ \\ aiholgueras@edu.uned.es \\ Universidad Nacional de Educación a Distancia, UNED, España
}

\section{Resumen:}

El objetivo principal de la investigación es conocer la importancia que tiene la orientación profesional para los jóvenes que retornan a la Educación Secundaria Obligatoria a través de los Centros de Educación de Personas Adultas, como herramienta indispensable que ha de estar presente en los momentos más críticos del estudiante atendiendo sus necesidades a nivel formativo, educativo y profesional, teniendo en cuenta los intereses, capacidades y necesidades de cada momento, así como la situación actual del mercado de trabajo, donde los jóvenes pasan con mayor rapidez entre situaciones de empleo y desempleo que en décadas anteriores.

La Metodología de Investigación se desarroIla a través de un análisis factorial exploratorio, realizado de acuerdo al procedimiento de los componentes principales, analizando una muestra total de 878 estudiantes matriculados en los cuatro cursos de Educación Secundaria Obligatoria en los Centros de Educación de Personas Adultas de la pro-

\begin{abstract}
:
The main objective of this piece of research is to understand the importance of career guidance for young people who return to compulsory secondary education through the Centers for Adult Education. Career guidance should be seen as an indispensable tool that must be present in students' most critical moments and whose main aims should be to focus on students' training needs, educational and professional level. Career guidance should also take into account the interests, abilities and needs of each moment and the current situation of the labor market in which young people, unlike in previous decades, are prone to have short-term jobs. The research methodology was developed through an exploratory factor analysis, performed according to the method of the main components, analyzing a total sample of 878 students enrolled in the four years of Compulsory Secondary Education at the Centers of Adult Education in Ciudad Real. Data were collected through the
\end{abstract}

1 Dirección para correspondencia (correspondence address):

Ana Isabel Holgueras González. Dpto. de Didáctica, Organización Escolar y Didácticas Especiales. Facultad de Educación. Universidad Nacional de Educación a Distancia, UNED. C/ Juan del Rosal, 10. 28040 Madrid (España). 
Análisis de la influencia de la orientación profesional en los jóvenes en situación de abandono escolar

Ana Isabel Holgueras González

vincia de Ciudad Real, datos recogidos a través de la cumplimentación de un cuestionario diseñado para tal efecto y donde los resultados de la investigación indican que el $84 \%$ de los alumnos encuestados opina que se necesita una orientación a lo largo de la vida para estar informado y motivado en todo momento, y el $75.6 \%$ considera que la orientación profesional es un instrumento que ayuda a la inserción profesional de los jóvenes. Tanto los que han acudido a un orientador profesional como los que no lo han hecho, confirman la importancia de la orientación: el $57.4 \%$ reconoce que no habría abandonado los estudios si hubiera recibido una adecuada orientación profesional y el $61.6 \%$ confirma que cuando finalice la Educación Secundaria Obligatoria acudirá a un orientador profesional para que le ayude en la búsqueda de empleo y el $74.7 \%$ para que le informe tanto de formación reglada como no reglada.

\section{Palabras clave:}

Orientación profesional; abandono escolar; medidas de segunda oportunidad; educación secundaria; Centros de Educación de Personas Adultas. completion of a questionnaire designed for this purpose and research results indicate that $84 \%$ of students surveyed believe that guidance throughout life needs to be informed and motivated at all times, and $75.6 \%$ believe that career guidance is an instrument that helps the employability of young people. Both those students who have gone to a professional counselor and those who have not, confirm the importance of guidance: $57.4 \%$ admit that they would not have dropped out had they received adequate vocational guidance and $61.6 \%$ confirm that when they finish Secondary Education they will visit a professional counselor to ask for help in finding employment and $74.7 \%$ of students will do the same in order to obtain information about formal and informal education possibilities.

\section{Keywords:}

Career counseling; dropout; second chance measures; secondary education; adult center.

\section{Résumé:}

L'objectif principal de la recherche est de comprendre l'importance de l'orientation professionnelle pour les jeunes qui retournent à l'enseignement secondaire obligatoire à travers les centres d'éducation pour adultes, comme un outil indispensable qui doit être présent dans les moments les plus critiques des étudiants et qui répondent à leurs besoins de formation, à leur niveau éducatif et professionnel, en tenant compte des intérêts, des aptitudes et des besoins du moment et de la situation actuelle du marché du travail, où les jeunes passent plus rapidement à des situations dsemploi et de chômage que dans les décennies précédentes.

La méthodologie de recherche a été développée par une analyse factorielle exploratoire, réalisée selon la méthode des composantes principales, analyse diun échantillon total de 878 étudiants inscrits dans les quatre années des centres d`enseignement secondaire de l séducation des adultes Ciudad Real, les données recueillies grâce à lıachèvement d`un questionnaire conçu à cet effet et où les résultats de la recherche indiquent que $84 \%$ des étudiants interrogés croient qu'une orientation tout au long de la vie est nécessaire pour être informé et motivé à tout moment, et 75,6\% estiment que lıorientation professionnelle est un instrument qui aide à lıemployabilité des jeunes. Aussi bien ceux qui sont allés voir un conseiller professionnel que ceux qui ne l`ont pas fait, confirment lıimportance de lıorientation: $57,4 \%$ admettent qu'ils n'auraient pas abandonné s'ils avaient reçu une orientation professionnelle adéquate et $61,6 \%$ confirment que lorsqu'ils auront terminé l'Enseignement secondaire ils iront voir un conseiller professionnel pour les aider à trouver un emploi et 74,7\% pour s'informer sur la formation formelle et l>apprentissage informel.

\section{Mots clés:}

Orientation professionnelle; abandon; mesures de la deuxième chance; enseignement secondaire; centre d'éducation des adultes. 
Fecha de recepción: 11-7-2015

Fecha de aceptación: 23-11-2015

\section{Introducción}

La calidad del sistema educativo de un país no sólo es indicador de sus niveles de desarrollo y de bienestar actuales, sino también venideros. Proveer de una escolaridad universal que además sea capaz de estimular el desarrollo integral de los jóvenes, es un objetivo estratégico de primera magnitud en las naciones más avanzadas, y debido a este abandono prematuro del sistema educativo tienen una baja confianza en sus posibilidades y una baja motivación para incorporarse a cualquier actividad, de forma especial, a programas formativos, lo que, unido a la dificultad de adaptarse a las crecientes exigencias laborales hace que aumente el riesgo de marginación económica y social. Como vemos, el problema del fracaso escolar no es sólo un problema educativo, sino que es también un problema con grandes repercusiones individuales y sociales. El mantenimiento de altas tasas de fracaso escolar provoca grandes y graves consecuencias en los individuos y en la sociedad.

En este entorno, la orientación profesional, debe aparecer como un poderoso arma para luchar contra el fracaso escolar, lo que hace necesario, que dicha orientación salga de los límites convencionales en los que se encuadran la mayoría de sus actividades, para conseguir una orientación encaminada a acciones y medidas de información, motivación, asesoramiento y acompañamiento a lo largo de todo el proceso de formación-empleo del joven; itinerario orientativo que incluso puede durar años. No debe adoptar exclusivamente el rol de parchear el mal funcionamiento del sistema educativo y del mercado de trabajo, sino que debe adoptar un carácter preventivo, entendiéndose en términos de proceso y cambio y buscando, de forma especial, la integración entre los sistemas educativo y productivo.

En la actualidad, motivado de forma especial por la falta de empleo, muchas de las personas que no titularon en su día en Educación Secundaria Obligatoria, deciden obtener el título y una de las vías más habitual es a través de la matriculación en un Centro de Educación de Adultos. La Ley 23/2002, de 21 de noviembre de 2002, de Educación de Personas Adultas de Castilla La Mancha, tiene entre sus objetivos el 
Análisis de la influencia de la orientación profesional en los jóvenes en situación de abandono escolar

Ana Isabel Holgueras González

de facilitar el desarrollo de las capacidades intelectuales y afectivas que posibiliten la formación integral de la persona, el aprendizaje autónomo, la adaptación a los cambios sociales y la participación activa en la sociedad de las personas adultas.

\section{Orientación profesional y medidas de segunda oportunidad para el retorno de los jóvenes a la educación}

En el momento actual del panorama español y europeo, la orientación profesional se entiende como un apoyo proactivo a los ciudadanos, y se está convirtiendo en uno de los ejes centrales de las políticas activas de empleo, así como en una base desde la cual evaluar necesidades, planificar las acciones de formación y evaluar los resultados. De esta manera, el aprendizaje a lo largo de la vida se integra con la orientación a lo largo de la vida, como claves para el empleo. Cada vez cobran más sentido las estrategias encaminadas a ayudar a los ciudadanos a desarrollar su proyecto profesional, con el fin de propiciar un "punto de encuentro" entre las exigencias del propio individuo y las oportunidades externas, proyecto profesional que aparece como eje vertebrador de la actividad orientadora, en tanto que permite al individuo definir o redefinir en cada momento, clarificar su identidad y planificar sus itinerarios de forma correcta. La orientación profesional es un proceso flexible, no estanco, ya que orientador y usuario decidirán cuando hay que avanzar, en qué sentido y cuándo se da por terminada la acción.

En materia educativa, la Estrategia de Lisboa (2000) fijó cinco objetivos, de los cuales, cuatro de ellos de forma directa y uno indirectamente hacían referencia a la necesidad de reducir los altos índices de fracaso escolar; se establece la necesidad de llegar a 2010 con una tasa de abandono escolar temprano igual o inferior al $10 \%$, así como la necesidad de incrementar hasta el $85 \%$ el porcentaje de jóvenes que completaran la educación secundaria. También se marcaba la recomendación de alcanzar el $12.5 \%$ de participación de la población en programas de educación permanente. Teniendo como base dicho indicador y utilizado como punto de referencia para el seguimiento de los Objetivos 2010-2020 de los sistemas educativos y formativos, se debe considerar en situación de abandono educativo temprano a todas aquellas personas que han salido del sistema educativo sin tener la cualificación necesaria para desenvol- 
verse en una sociedad tecnificada y moderna, independientemente de su edad, así como todos aquellos ciudadanos que, estando escolarizados y no habiendo abandonado el sistema educativo, son población de riesgo (Plan para la reducción del abandono educativo temprano 2014-2020).

Aunque el problema no es nuevo, adquiere especial importancia en la sociedad en la que vivimos, ya que el conocimiento es clave para el desarrollo económico, pero también para que la persona pueda acceder a un trabajo y a un estilo de vida digno y como vemos, dicho problema tiene una doble vertiente: educativa, ligada al fracaso escolar y al abandono del sistema, y social, como merma de las posibilidades de empleo, aumento de las posibilidades de marginación, paro, etc.

La escuela no puede seguir pensando que los alumnos van a enfrentarse, cuando terminen la etapa básica de sus estudios, a una sociedad homogénea y estable, sino más bien a una sociedad heterogénea, cambiante e imprevisible. Todo esto plantea consecuencias importantes para el alumnado que fracasa en su aprendizaje, que habitualmente encuentra dificultades para la formación así como falta de habilidades socio afectivas y personales, por lo que los programas que busquen la superación de sus problemas han de tener en cuenta ambas dimensiones, contando con los cambios que se producen en las nuevas generaciones, ya que el alumnado actual, desde que nace, vive en una sociedad audiovisual e informatizada, en la que la rapidez de la información, la capacidad de selección y el predominio del elemento visual están modificando los estilos de atención y sus estrategias de aprendizaje. Los jóvenes tienen que adaptar la enseñanza a sus características y conectar con su manera de situarse en la vida.

Según las últimas investigaciones, "el alumno absentista comparado con el alumno no absentista, desarrolla niveles inferiores de autoconcepto académico, autoestima y competencia social en el aula, y además otorgan menos valor al control parental, sus relaciones familiares suelen ser más conflictivas y el estilo educativo predominante en su entorno familiar es el permisivo. Habitualmente, este tipo de alumnos no acata normas ni asume responsabilidades y suele tener problemas de comportamiento, atención y razonamiento". (Garfaella, Gargallo y Sánchez, 2001). Concretando datos, casi la tercera parte del alumnado de Educación Secundaria Obligatoria obtiene calificaciones negativas: un $32 \%$ repite curso y un $35 \%$ no finaliza $2^{\circ}$ curso con éxito. La primera consecuencia de estos resultados es que el $72 \%$ de la desocupación de 
Análisis de la influencia de la orientación profesional en los jóvenes en situación de abandono escolar

Ana Isabel Holgueras González

los menores de 25 años tiene relación directa con el abandono de los estudios y el fracaso escolar. Uno de cada cuatro jóvenes fracasa en sus estudios, con los consiguientes problemas que comporta, de índole familiar, psicológica y en su futura formación profesional e inserción laboral. (Instituto Nacional de Calidad y Evaluación, 2015).

El fracaso escolar es una situación polivalente y compleja, y en el momento de la intervención hay que tener en cuenta diversas variables. Es un trabajo de equipo y no se circunscribe de forma exclusiva al ámbito escolar. De hecho, para buscar soluciones eficaces, debería haber participación por parte de los distintos agentes sociales y educativos de la red de intervención del territorio, por lo que se hace necesaria una coordinación que articule las diversas actuaciones, y que tenga en cuenta unos objetivos socioeducativos comunes, consensuados por todos los que pueden intervenir con el colectivo de jóvenes en situación de fracaso escolar, en consecuencia, si se lograra una reducción del fracaso escolar, esto posibilitaría un aumento de la cohesión social, se reafirmaría el derecho de todos los ciudadanos y de forma especial el de los más jóvenes, a la educación, y se frenarían los mecanismos generadores de la exclusión educativa.

El Plan para la reducción del abandono educativo temprano 20142020, tiene como principal fortaleza la cooperación de las Administraciones Educativas, lo que implica extender el acceso a la información, orientación y asesoramiento académico y profesional de calidad, desarrollar medidas de prevención, intervención y compensación para reducir el abandono educativo temprano, sensibilizar y formar a los profesionales, flexibilizar las formas de acceso a la educación y a la formación, así como garantizar sistemas de reconocimiento de las competencias propias de las enseñanzas obligatorias y de perfiles profesionales, entre otras. Medidas que se estructuran de acuerdo con las indicaciones de la Unión Europea en tres categorías: prevención, intervención y compensación, siendo a través de esta última donde se proponen medidas de compensación que tratan de promover el retorno de aquellos que abandonaron los procesos formativos antes de alcanzar la cualificación necesaria; las actuaciones van encaminadas a ofrecer una segunda oportunidad al esfuerzo y la capacidad del alumnado favoreciendo su vuelta al sistema educativo para mejorar su educación y aumentar las posibilidades de acceso al mercado laboral, en algunos casos se instituye la figura del asesor personal o tutor de referencia que estimula, aconseja 
y asesora al ciudadano para que adquiera los hábitos necesarios para poder participar satisfactoriamente en actividades formativas. (Plan para la reducción del abandono educativo temprano, 2014).

Es importante tener en cuenta que la educación de personas adultas es un componente fundamental del aprendizaje permanente que se dirige a quienes han superado la edad máxima de escolarización obligatoria, los 18 años, tienen 16 años y disponen de contrato laboral o son deportistas de alto rendimiento. Esta educación secundaria para personas adultas, permite obtener el Graduado en Educación Secundaria Obligatoria en dos años a través de las modalidades presencial o a distancia, esta última tanto ordinaria como en teleformación. También existe la posibilidad de presentarse a una prueba libre si se es mayor de 18 años, bien a través de la educación de personas adultas o bien a través de una prueba extraordinaria en los centros de enseñanza secundaria donde el alumnado ha realizado sus estudios, siempre y cuando no tenga más de cinco materias pendientes. Además, destacar que existe formación orientada al mundo laboral en educación de personas adultas, que comprende dos tipos de acciones: formación profesional asociada a títulos y enseñanzas no formales de formación profesional, y que ambas ofrecen la posibilidad de mejorar sus cualificación profesional, adquirir una preparación para el ejercicio de otras profesiones y disponer de los recursos necesarios para adquirir los objetivos de la formación profesional. Esta formación se organiza con una metodología flexible y abierta, basada en el autoaprendizaje, de tal modo que permita conciliar el aprendizaje con otras actividades.

\section{Método}

\section{Objetivos}

La investigación ha pretendido comprobar la necesidad de la orientación profesional a lo largo de la vida en aquellos jóvenes que abandonaron el sistema educativo y retoman a la educación a través de los Centros de Educación de Adultos, planteándose los siguientes objetivos específicos:

1. Conocer si los Institutos de Educación Secundaria ofrecen información y orientación suficiente a los estudiantes sobre las distintas opciones educativas.

2. Averiguar si el alumnado que abandona de forma prematura la 
Análisis de la influencia de la orientación profesional en los jóvenes en situación de abandono escolar

ana Isabel Holgueras González

educación se sentiría más motivado para retornar si recibiera una formación más flexible, creativa, colaborativa y práctica.

3. Conocer si el desarrollo de un itinerario orientativo individualizado amplía las posibilidades de reincorporación de los jóvenes que en su día abandonaron la educación obligatoria.

\section{Diseño de la investigación}

En el estudio que nos ocupa se ha elegido un diseño de investigación no experimental transversal, se analizan los datos observando situaciones ya existentes sin manipular las variables, sin que exista un control directo ni se pueda influir sobre ellas, la realidad ya está diseñada y sólo se analiza en un momento del tiempo determinado, sin analizar su evolución posterior. Se desea analizar los itinerarios orientativos a los que acceden los jóvenes que abandonaron la ESO sin titular y actualmente estudian en un Centro de Educación de Adultos, si conocen y utilizan la orientación profesional, y cómo mejorar dichos itinerarios para reducir el número de abandonos, pero en un momento determinado, no se modifican las variables para comprobar cómo reacciona un grupo, por ejemplo, ni se analizan en dos períodos de tiempo distintos para analizar la existencia de modificaciones.

\section{Técnicas e instrumentos para la recogida de datos.}

Se utilizan técnicas cuantitativas a través de un cuestionario dirigido a estudiantes matriculados en los cuatro cursos de Educación Secundaria Obligatoria en los Centros de Educación de Adultos de la provincia de Ciudad Real. Se realizan un total de 65 preguntas cortas de respuestas cerradas en una escala Likert ( 1 muy en desacuerdo/5 muy de acuerdo).

Las variables se clasifican en cinco dimensiones:

- Datos identificativos

- Educación-formación

- Orientación

- Empleo

- Abandono escolar

Utilizamos dos estrategias para comprobar la validez, por una parte, la consulta a expertos (orientadores y profesores de Institutos de Edu- 
cación Secundaria y de Universidad) y por otra, una aplicación piloto (Creswell, 2002), con la idea de que se realizaran las observaciones oportunas sobre la adecuación, claridad, formato, número de ítems, así como cualquier otra cuestión necesaria para una mejor comprensión del cuestionario por parte de los estudiantes.

Consulta a Expertos: la necesidad de disponer de un instrumento de recogida de datos fiable y de calidad, nos lleva a utilizar la técnica de juicio de expertos, que consiste en preguntar a uno o varios expertos si los reactivos evalúan lo que se quiere que evalúen (Salkind, 1999). En la investigación, los expertos pertenecen tanto al ámbito de la orientación (orientadores de Institutos de Enseñanza Secundaria, orientadores de oficinas de empleo y orientadores de Centros de Adultos) como al ámbito docente (profesores de Institutos, de Centros de Adultos y profesores universitarios); un número total de diez expertos.

La otra estrategia que utilizamos para calcular la validez es la realización de una Prueba Previa Piloto, procedimiento por el cual el investigador puede realizar cambios en el cuestionario, si son necesarios, basándose en el feedback proporcionado por un pequeño número de individuos a los que se les entrega el cuestionario y lo completan. Realizan el cuestionario 30 estudiantes matriculados en el Centro de Adultos de La Solana (Ciudad Real) y se observa que hay cinco preguntas que la mayoría no comprenden su significado, por lo que se procede a cambiar la formulación de las mismas para hacerlas más sencillas a su lectura.

Tanto la valoración de los expertos como la prueba piloto nos ayudaron enormemente a la hora de redactar un instrumento de recogida de datos más fiable que el inicialmente propuesto. El procedimiento seleccionado para administrar y recoger el instrumento de investigación, en este caso, el cuestionario del alumnado matriculado en los Centros de Educación de Adultos es la entrega y recogida de forma presencial.

El cálculo de la fiabilidad del cuestionario se lleva a cabo mediante el procedimiento del Alfa de Cronbach, el cual revela el siguiente resultado: 0,812 .

\section{Descripción de la población y muestra}

La población a la que nos dirigimos para aplicar los cuestionarios son los estudiantes matriculados en cualquiera de los cuatro cursos en los Centros de Educación de Adultos de la provincia de Ciudad Real; como 
Análisis de la influencia de la orientación profesional en los jóvenes en situación de abandono escolar

Ana Isabel Holgueras González

se realizan de forma presencial a los distintos grupos y explicando los objetivos de la investigación, y se recogen en el mismo acto, se cumplimentan todos los que se entregan, un total de 878 repartidos en: Alcázar de San Juan, Almadén, Ciudad Real, Daimiel, La Solana, Manzanares, Piedrabuena, Puertollano, Tomelloso, Valdepeñas y Villanueva de los Infantes.

Distribuidos como sigue:

Tabla 1. Centros de Educación de Adultos participantes.

\begin{tabular}{|ll|}
\hline Centros de Adultos & No cuestionarios \\
\hline Alcázar de San Juan & 88 \\
Almadén & 32 \\
Ciudad Real & 178 \\
Daimiel & 78 \\
La Solana & 51 \\
Manzanares & 83 \\
Piedrabuena & 48 \\
Puertollano & 127 \\
Tomelloso & 73 \\
Valdepeñas & 91 \\
Villanueva de los Infantes & 29 \\
Total & $\mathbf{8 7 8}$ \\
\hline
\end{tabular}

En la comunidad de Castilla La Mancha hay un total de 33 Centros de Educación de Adultos repartidos de la siguiente forma: Albacete: 5, Ciudad Real: 11, Cuenca: 4, Guadalajara: 3 y Toledo: 10.

Tabla 2. Respuestas por curso.

\begin{tabular}{|ll|}
\hline Curso de Educación Secundaria Obligatoria & Respuestas \\
\hline Primero & $114(13 \%)$ \\
Segundo & $202(23 \%)$ \\
Tercero & $258(29,4 \%)$ \\
Cuarto & $304(34,6 \%)$ \\
Total & $\mathbf{8 7 8}(\mathbf{1 0 0} \%)$ \\
\hline
\end{tabular}

Tabla 3. Género.

\begin{tabular}{|ll|}
\hline Género & Número \\
Hombres & $476(54,21 \%)$ \\
Mujeres & $402(45,79 \%)$ \\
Total & $\mathbf{8 7 8}(\mathbf{1 0 0} \%)$ \\
\hline
\end{tabular}




\section{Resultados}

Una vez recogidos los datos, se procede a su codificación informática con la ayuda del paquete informático SPSS 17.0.

Respecto al apartado educación/formación, destacamos que la inmensa mayoría de los encuestados son conocedores de la importancia que tiene la formación en la sociedad actual, a través de respuestas como: el $64.7 \%$ está muy de acuerdo en que es bueno formarse en una época de crisis, el $86.2 \%$ está muy de acuerdo o de acuerdo en que la formación facilita el acceso al mercado de trabajo, el 61.7\% está muy en desacuerdo o en desacuerdo en que no es necesario estudiar para conseguir un buen empleo, así como que el $72 \%$ considera que la falta de formación dificulta la búsqueda de empleo.

Si hablamos de metodología didáctica y nuevas tecnologías, el 79.4\% aprenden mejor con una metodología creativa y el $81.7 \%$ con clases más prácticas y participativas. El $78.3 \%$ consideran que las nuevas tecnologías facilitan el aprendizaje en el aula y que es necesario tener conocimientos básicos de informática e Internet para buscar empleo: el $71.6 \%$. Respecto a la metodología docente de los profesores de los Centros de Educación de Adultos, al $72.7 \%$ le gusta y el $76 \%$ afirma que los profesores conocen y utilizan las nuevas tecnologías.

En el apartado de orientación, más de la mitad de los estudiantes (57.4\%) coinciden en que si hubieran tenido una buena orientación no hubieran abandonado los estudios, y el $67 \%$ y el $74.7 \%$ acudirá a un orientador a que le informe y asesore en la búsqueda de empleo así como en todo lo relacionado con la formación. El 46\% confirma conocer el servicio de orientación de las oficinas de empleo y el $40 \%$ afirma que fue este orientador el que les informó de la existencia del Centro de Educación de Adultos. Es reveladora la respuesta del 84\% de los encuestados que dicen estar muy de acuerdo o de acuerdo en que se necesita una orientación a lo largo de la vida para estar informados y motivados en todo momento, así como la del $75.6 \%$ que considera que la orientación profesional es un instrumento que ayuda a los jóvenes en su inserción laboral.

Cuando analizamos el apartado de empleo, se llega a la conclusión de que el $74.2 \%$ de los jóvenes encuestados, consideran que sin titulación académica existe desigualdad en el acceso al empleo y a la sociedad; un $77.4 \%$ afirman que cuando se reactive el mercado de trabajo 
Análisis de la influencia de la orientación profesional en los jóvenes en situación de abandono escolar

Ana Isabel Holgueras González

se necesitará personal cualificado y respecto a los recursos que ofrece la Administración en relación a la formación y al empleo, casi el 32\% confirma que los desconoce, frente a un $44.3 \%$ que dice sí conocerlos.

En el análisis del apartado varios, destacar lo que sigue:

- El 89.5\% tiene ordenador en su domicilio, el 82\% conexión a Internet y el $89.7 \%$ conoce y utiliza las nuevas tecnologías habitualmente.

- El $64.5 \%$ ha realizado en alguna ocasión cursos de formación.

- El 29.5\% se ha matriculado en un Centro de Educación de Adultos porque le informó y asesoró un orientador.

- El $46 \%$ abandonó el Instituto porque empezó a trabajar y un $22 \%$ porque se aburría en clase.

- El $47 \%$ ha vuelto a estudiar porque considera que es necesario formarse, el 25\% porque no encuentra trabajo sin el título de Educación Secundaria Obligatoria y el 21\% porque prefiere estudiar a estar en casa sin hacer nada.

- Cuando estos jóvenes finalicen la Educación Secundaria Obligatoria, el 33\% buscará empleo y el mismo porcentaje continuará en la formación reglada, seguido del $29 \%$ que realizará cursos de especialización.

- La modalidad de estudio que prefieren estos jóvenes es presencial $(68.8 \%)$, seguida de mixta $(18.9 \%)$ y a distancia en último lugar $(12.3 \%)$.

Se resume la información en tablas, midiendo la asociación entre las dos variables que forman dicha tabla, en este caso género y motivos por los que abandonó el Instituto, género y ¿qué hará cuando finalice la ESO? y curso y género y modalidad preferente de estudio. 
Tabla 4. Género y Motivos por los que abandonó el Instituto.

\begin{tabular}{|c|c|c|c|c|c|c|c|c|}
\hline & & \multicolumn{7}{|c|}{ Abandoné el Instituto por } \\
\hline & & $\begin{array}{c}\text { Me } \\
\text { aburría } \\
\text { en } \\
\text { clase }\end{array}$ & $\begin{array}{c}\text { Empecé } \\
\text { a } \\
\text { trabajar }\end{array}$ & $\begin{array}{l}\text { Tuve } \\
\text { un } \\
\text { hijo }\end{array}$ & $\begin{array}{c}\text { Movilidad } \\
\text { familiar }\end{array}$ & $\begin{array}{c}\text { Problemas } \\
\text { de relación } \\
\text { con el } \\
\text { profesorado }\end{array}$ & $\begin{array}{c}\text { No lo } \\
\text { conside- } \\
\text { raba } \\
\text { necesario }\end{array}$ & Otro \\
\hline \multirow{2}{*}{ sexo } & Hombre & 106 & 232 & 2 & 8 & 42 & 40 & 46 \\
\hline & Mujer & 84 & 172 & 10 & 8 & 18 & 54 & 56 \\
\hline Total & & 190 & 404 & 12 & 16 & 60 & 94 & 102 \\
\hline
\end{tabular}

Destacar que el $46 \%$ de los jóvenes abandonó los estudios porque encontraron empleo, de los cuales el $74 \%$ fueron hombres, seguido del $24 \%$ que abandonaron porque se aburrían en clase, siendo también, mayoría de género masculino.

Tabla 5. Género y ¿Qué haré cuando finalice la ESO?

\section{Cuando finalice la ESO}

\begin{tabular}{lccccccc}
\cline { 3 - 6 } $\begin{array}{l}\text { Buscaré } \\
\text { empleo }\end{array}$ & $\begin{array}{c}\text { Seguiré } \\
\text { estudiando especialización }\end{array}$ & $\begin{array}{c}\text { Haré cursos de } \\
\text { empleo ni } \\
\text { formación }\end{array}$ & Otro & $\begin{array}{c}\text { Buscaré Total } \\
\text { empleo }\end{array}$ & \\
\hline \multirow{2}{*}{ sexo Hombre } & 168 & 152 & 138 & 8 & 10 & 476 \\
& 126 & 138 & 116 & 2 & 20 & 402 \\
\hline Mujer & 126 & $\mathbf{2 9 0}$ & $\mathbf{2 5 4}$ & $\mathbf{1 0}$ & $\mathbf{3 0}$ & $\mathbf{8 7 8}$ \\
\hline
\end{tabular}

Tan sólo 2 mujeres y 8 hombres no buscarán empleo ni formación cuando obtengan el título de Educación Secundaria Obligatoria, y aunque los porcentajes están bastante igualados, son mayoría el número de hombres que buscarán empleo o seguirán estudiando.

Tabla 6. Curso y Modalidad preferente de estudio.

\begin{tabular}{lccccc}
\hline \multirow{2}{*}{ Presencial } & \multicolumn{3}{c}{ Prefiero estudiar en modalidad } & \multirow{2}{*}{ Total } \\
\cline { 3 - 5 } & & A distancia & Mixta & Presencial & \\
\hline \multirow{4}{*}{ curso } & 1 ESO & 90 & 8 & 16 & 114 \\
& 2 ESO & 146 & 18 & 38 & 202 \\
& 3 ESO & 178 & 34 & 46 & 258 \\
\cline { 3 - 5 } Total & 4 ESO & 190 & 48 & 66 & 304 \\
\hline
\end{tabular}


Análisis de la influencia de la orientación profesional en los jóvenes en situación de abandono escolar

Ana Isabel Holgueras González

El $69 \%$ de los estudiantes para su aprendizaje prefieren asistir a clase antes que recibir formación a distancia, lo que resulta más evidente en los estudiantes de últimos cursos $\left(3^{\circ}\right.$ y $\left.4^{\circ}\right)$.

Tabla 7. Género y modalidad preferente de estudio.

\begin{tabular}{lccccc}
\hline & \multicolumn{3}{c}{ Prefiero estudiar en modalidad } & \\
\cline { 2 - 4 } \multicolumn{2}{l}{ Presencial } & A distancia & Mixta & Presencial & Total \\
\hline sexo & Hombre & 310 & 74 & 92 & 476 \\
& Mujer & 294 & 34 & 74 & 402 \\
\hline Total & $\mathbf{6 0 4}$ & $\mathbf{1 0 8}$ & $\mathbf{1 6 6}$ & $\mathbf{8 7 8}$ \\
\hline
\end{tabular}

Teniendo en cuenta el género, los resultados son muy similares: el 51\% de los hombres prefieren la modalidad presencial así como el $49 \%$ de las mujeres preguntadas.

La Técnica del Análisis de la Varianza (ANOVA) es un método muy flexible que nos permite dividir la varianza de la variable dependiente en dos o más componentes, y que, en nuestro caso, se realiza el análisis de la varianza de un factor, seleccionando variables dependientes (de una en una) y una sola variable de factor independiente que es el género.

\section{ANOVA}

Tabla 8. Motivos de la vuelta a la educación.

\begin{tabular}{lccccr}
\hline \multicolumn{6}{c}{ He vuelto a estudiar porque } \\
\hline & Suma de cuadrados & gl & Media cuadrática & F & Sig. \\
\hline Inter-grupos & 74,658 & 1 & 74,658 & 23,850 &, 000 \\
Intra-grupos & 2742,198 & 876 & 3,130 & & \\
\hline Total & $\mathbf{2 8 1 6 , 8 5 6}$ & $\mathbf{8 7 7}$ & & & \\
\hline
\end{tabular}

Se lleva a cabo un análisis factorial exploratorio, realizado de acuerdo al procedimiento de los componentes principales; no se establecen "a priori" los factores, sino que se pretende que éstos se determinen mediante el propio análisis. También se efectúa una rotación oblicua, que es la más utilizada en estos casos para ofrecer resultados más claros y que, además, nos aporta información sobre la relación entre factores.

Las variables utilizadas se adecúan a un modelo de análisis de factores, en concreto de 15 factores, que explican el $60.345 \%$ de la varianza total explicada. 
Tabla 9. Factores.

\begin{tabular}{lll}
\hline Factor & Ítems & Variables \\
\hline Factor 1 & $11,12,14,15,16,25,26$ & Metodología creativa \\
\hline Factor 2 & $1,5,6,7,22,23$ & Necesidad de la formación \\
\hline Factor 3 & $35,36,39,40$ & Orientación a lo largo de la vida \\
\hline Factor 4 & $43,44,45,49,50$ & Acceso al mercado de trabajo \\
\hline Factor 5 & $30,31,34,38$ & Orientación educativa \\
\hline Factor 6 & $9,41,42,47$ & Desigualdad laboral \\
\hline Factor 7 & $2,4,13$ & Combinación formación-empleo \\
\hline Factor 8 & 8,21 & Plazas en los Centros de Formación de Adultos \\
\hline Factor 9 & 33 & Orientación profesional \\
\hline Factor 10 & $10,29,48$ & Estudiar para trabajar \\
\hline Factor 11 & $17,19,24$ & Encontrar empleo con la ESO \\
\hline Factor 12 & 20,28 & Sin ESO no hay formación \\
\hline Factor 13 & 18,27 & Uso de las Nuevas Tecnologías en el aula \\
\hline Factor 14 & 37 & Calidad de la orientación \\
\hline Factor 15 & 3,46 & Recursos de la Administración \\
\hline
\end{tabular}

\section{Conclusiones}

En la actualidad, no ponemos en duda la importancia de la orientación profesional para mejorar las tasas de fracaso escolar y favorecer una mejor elección de los estudios y/o profesiones, por lo que se hace necesario proponer acciones concretas en dos ámbitos: el educativo y el laboral, motivo por el que podemos ver a los orientadores como "reorientadores" de la carrera profesional. Es el momento de actuar, ya que el objetivo principal es que aquellos estudiantes que no quisieron o no pudieron seguir estudiando, lo hagan posteriormente a través de las distintas opciones o itinerarios que ofrece tanto el sistema reglado como el no reglado.

El planteamiento educativo actual, que intenta responder a las necesidades de una sociedad desarrollada, multicultural y abierta, y en el que destacan los aspectos más formativos y personalizadores de la educación, pone de manifiesto la necesidad de un modelo de orientación que llegue a todos y que se integre plenamente en la actividad educativa de estos jóvenes que retoman su formación y de forma especial, porque la inserción juvenil en el mercado laboral se está convirtiendo, cada día más, en un proceso prolongado, por lo que, la formación se vuelve una herramienta indispensable para estos jóvenes y hace que la responsabi- 
Análisis de la influencia de la orientación profesional en los jóvenes en situación de abandono escolar

Ana Isabel Holgueras González

lidad de la orientación no sólo recaiga en el sector de educación, sino también en los servicios públicos de empleo, que deben orientar y proponer acciones formativas que permitan una inserción laboral adecuada.

Tal y como reclama la estrategia Europa 2020, ha de existir un marco estratégico que comprometa al sistema educativo en la consecución de los objetivos del empleo, a través de la reducción del fracaso escolar, la mejora de la orientación profesional y la garantía de las competencias clave para el trabajo, por lo que, entendemos que se hace necesario coordinar las políticas de empleabilidad entre las administraciones laborales y educativas cuanto antes.

Comenzamos nuestra investigación con una fase inicial que perseguía como objetivo conocer la opinión de los jóvenes que retornan a la educación a través de los Centros de Educación de Adultos en relación a distintas hipótesis planteadas al inicio del estudio. Respecto al objetivo principal, se corrobora la importancia de la orientación profesional, destacando las siguientes respuestas, el 57.4\% no habría abandonado los estudios si hubiera recibido una buena orientación profesional, el 84\% considera que se necesita orientación a lo largo de la vida para estar informado y motivado en todo momento, al $40.78 \%$ le informó un orientador profesional de la existencia de los Centros de Educación de Adultos, el $61.6 \%$ acudirá a un orientador para que le ayude en la búsqueda de empleo y el $74.7 \%$ para que le informe de educación y/o formación.

Los resultados confirman las conclusiones de la investigación llevada a cabo en 2009 por el Dr. García Brocello en la tesis doctoral Diseño y validación de un programa de orientación profesional para alumnos de E.S.O. en entornos rurales, en la que indica que es imprescindible lograr una mayor presencia de la orientación profesional, de forma especial en los estudiantes en riesgo de abandono del sistema educativo, porque la mayor parte de actividades de orientación profesional recogidas en el plan de acción tutorial, son de tipo informativo, careciendo, por tanto, de atención integral y los tutores no se sienten implicados en el diseño y desarrollo de actividades recogidas en el plan de acción tutorial entre las que se encuentran las actividades relacionadas con la orientación profesional. Los estudiantes valoran la necesidad de aplicación de programas de orientación profesional y que dichos programas sean aplicados por agentes externos. También se confirman los estudios de Watts y Kidd, 2000, cuando destacan que la Orientación Profesional en lugar de centrarse en ayudar a los individuos a elegir una profesión se debe 
centrar en ayudarles a construir la carrera profesional. La carrera profesional ha de definirse desde la preadolescencia y adolescencia por un proceso de decisiones múltiples, por una secuencia de microdecisiones. Se debe diseñar una nueva práctica de la intervención orientadora en las diversas etapas de la vida (etapa de decisión académica y de elección de itinerarios formativos, etapa de inserción laboral, etapa de adaptación o cambios en el profesional adulto, reorientación y reinserción en algunos momentos de la trayectoria laboral, e incluso, en la preparación para la jubilación. (Corominas, 2006).

En relación a los objetivos específicos, los estudiantes indican que no conocen los recursos que ofrece la Administración Pública respecto a formación y empleo (31.7\%), el 79.4\% considera que aprenden mejor con una metodología didáctica creativa, el $81.7 \%$ prefiere clases prácticas y participativas y un $78.3 \%$ dice estar de acuerdo en que el uso de las nuevas tecnologías en el aula facilita el aprendizaje, esto último coincidente con los resultados de un estudio realizado por la autora, denominado La formación docente y la figura del formador ocupacional, a través del cual todas las partes implicadas (estudiantes, formadores y técnicos de formación) concluyen en la importancia y necesidad del uso de las nuevas tecnologías en el aula que enriquecen la metodología didáctica facilitando a su vez la comprensión de la materia y la participación de los estudiantes. Además, el $86.2 \%$ piensa que la formación facilita el acceso al mercado de trabajo, tan sólo un $17.81 \%$ indica que el orientador del Instituto le informó de la situación del mercado de trabajo y el 75\% opina que la orientación profesional es una herramienta de ayuda a la inserción profesional de los jóvenes. Finalmente indicar que el principal motivo por el que los jóvenes retornan al sistema educativo es porque consideran necesario formarse (47\%), de forma especial en esta época de crisis, aunque también porque sin el Título de Educación Secundaria Obligatoria es más difícil encontrar empleo (25\%).

Para concluir, destacar que la orientación profesional debe abarcar un significado global y transversal, que permita "acompañar" a la persona en la planificación de su carrera a lo largo de toda la vida académica y profesional, no limitándose a los estudiantes que fracasan en el sistema educativo, sino ofreciendo atención también a los que superan los objetivos académicos y no sólo desde la orientación educativa que frecuentemente se limita a ofrecer información a los estudiantes y a ayudarles a resolver los problemas que la vida académica les plantea, sino desde la 
Análisis de la influencia de la orientación profesional en los jóvenes en situación de abandono escolar

Ana Isabel Holgueras González

orientación profesional que se lleva a cabo a través de un proceso continuo, global y holístico, que ha de estar presente en los momentos más críticos del estudiante atendiendo todas sus necesidades a nivel educativo, formativo y laboral, teniendo en cuenta los intereses y capacidades de cada momento, trabajando la motivación y la autoestima con el objetivo de mejora en el desarrollo personal y de toma de decisiones.

\section{Referencias bibliográficas}

ÁLVAREZ, M. (2006). La acción tutorial: su concepción y práctica. Bilbao. Ministerio de Educación y Ciencia.

CABERO, J. y MARTíNEZ, F. (1995) Nuevos canales de comunicación en la enseñanza. Madrid. Centro de Estudios Ramón Areces, S.A.

CANTÓN MAYO, I y Otros. (2015) Expectativas de los futuros profesores de Educación Secundaria. Enseñanza \& Teaching, 33. pp. 105-120.

CASTELL, M. (2001) La Galaxia Internet. Madrid. Plaza \&Janés.

CASTILLO, M. y otros (2006) ¿Cómo evitar el fracaso escolar en Secundaria? Recursos. Materiales 12-16 para enseñanza secundaria. Madrid. Ministerio de Educación y Ciencia. Narcea, S.A. de Ediciones.

CEDEFOP (2006). Mejorando las políticas y sistemas de orientación continúa. El uso de herramientas de referencias comunes en Europa. Luxemburgo: Oficina de Publicaciones Oficiales de las Comunidades Europeas.

CORBETTA, P. (2007) Metodología y Técnicas de Investigación Social. Bolonia. McGrawHill.

COROMINAS, E. (2006) Nuevas perspectivas de la orientación profesional para responder a los cambio y necesidades de la sociedad de hoy. Estudios sobre Educación, 2006, 11, 91-110.

CRESWELL, J. (2002). Educational research: Planning, conducting, and evaluating quantitative and qualitative research. Upper Saddle River, N.J: Merrill.

DE HARO, J.J. (2011) Manual Imprescindible de Redes Sociales para la Educación. Madrid. Ediciones Anaya Multimedia.

DE HARO, J.J. (2011) Redes sociales para la educación. Madrid. Anaya Multimedia.

GARCÍA, T. (2009) Diseño y validación de un programa de orientación profesional para alumnos de E.S.O. en entornos rurales. Tesis doctoral.

GARCÍA, L. (2007) De la educación a distancia a la educación virtual. Barcelona. Editorial Ariel S.A.

GARFAELLA, P.R y Otros (2001) Medidas y estrategias para la reducción del absentismo escolar. Revista de Estudios de Juventud 52, 27-36.

GODINO, C.M.B. (2006) Volver a la escuela: recorridos escolares adultos que desean culminar un trayecto. KAIRÓS. Revista de temas sociales. Año 10, nº18.

GOICOVIC, I. (2002) Educación, deserción escolar e integración laboral juvenil. Revista última Década. № 16. Marzo 2002. pp. 11-52. 
Análisis de la influencia de la orientación profesional en los jóvenes en situación de abandono escolar

ana Isabel Holgueras González

GONZÁLEZ, M. T., (2006) Absentismo Escolar: Una situación singular de la exclusión educativa. REICE- Revista Electrónica Iberoamericana sobre Calidad, Eficacia y Cambio en Educación. 2006, vol. 4, nº 1.

HERRERO, M. (2001) ¿Fracaso escolar o fracaso del sistema escolar? Revista de Estudios de Juventud, 2. pp. 59-63.

HOLGUERAS, A.I. (2011) La formación docente y la figura del formador ocupacional. Enseñanza \& Teaching, 29, 1, pp. 27-51.

INSTITUTO NACIONAL DE CALIDAD Y EVALUACIÓN. Informe 2015 sobre el estado del sistema educativo. Curso 2013-2014. Consejo Escolar del Estado. Secretaría General Técnica. Catálogo de Publicaciones del Ministerio: www.mecd.gob.es

MARCELO, C. y Otros (1995) Un instrumento para evaluar cambios en las etapas de preocupaciones de profesores: el inventario de preocupaciones de profesores. Ediciones Universidad de Salamanca. Enseñanza, 13, 1995, pp. 153-178.

MARCHESI, A. (2003) El fracaso escolar en España. Documento de trabajo 11/2003. Fundación Alternativas.

MEDINA, A. (2002) Formación y Empleo: capacitación de los educadores/as para la mejora de las personas adultas y las organizaciones productivas. Universidad Nacional de Educación a Distancia.

MEDINA, A. y SALVADOR, F. (2002) Didáctica General. Madrid. Pearson Educación.

ORTEGA, A. y COCA, M.A. (2004) Elaboración de un cuestionario para valorar la actitud del alumnado ante el uso de plataformas informativas. etic@net. Publicación en línea. Año II, número 3. Julio de 2004.

PLAN PARA LA REDUCCIÓN DEL ABANDONO EDUCATIVO TEMPRANO 2014-2020. Ministerio de Educación, cultura y deporte. Dirección General de Formación Profesional. Subdirección General de Aprendizaje a lo Largo de la Vida. www.educacion. gob.es

PÉREZ, V. y RODRÍGUEZ. J.C. (2002). La educación profesional en España. Madrid. Fundación Santillana.

RICOY, M.C. (2004) Claves para identificar el perfil del alumnado de la educación básica de adultos. Ediciones Universidad de Salamanca. Enseñanza, 22, 2004, pp. 283-300.

RODRÍGUEZ, S. (2007) La orientación educativa en una educación de calidad, en equidad. En Actas del I Congreso Internacional de Orientación Educativa de Andalucía. Vol. I pp. 159-189. Junta de Andalucía. Consejería de Educación: Dirección General de Participación y Solidaridad Ciudadana.

SALKIND J. (1999) Métodos de Investigación. Pearson Educación.

SEVILLANO, M.L. (2011) Educadores en Red. Madrid. Ediciones Académicas.

SEVILLANO, M.L. (coord.) (2008) Estrategias didácticas en el aula (buscando la calidad y la innovación). Madrid. UNED.

SEVILLANO, M.L. y VÁZQUEZ, E (2015) Modelos de Investigación en contextos ubicuos y móviles en Educación Superior, Madrid. MacGrawHill

SUREDA, I. (1998) Análisis del proceso de cambio de orientación en el contexto escolar de secundaria. Ediciones Universidad de Salamanca. Enseñanza, 16, 1998, pp. 297 311.

VAQUERO, A. (2005) El abandono escolar temprano en España: programas y acciones 
Análisis de la influencia de la orientación profesional en los jóvenes en situación de abandono escolar

Ana Isabel Holgueras González

para su reducción. Revista Galega do Ensino. Año 13, n47, noviembre de 2005.

VÁZQUEZ-CANO, E. (2011) Programar en Primaria y Secundaria. Madrid. Pearson Educación, S.A.

WATTS, A.G. y KIDD, J.M. (2000) Guidance in the United Kingdom: past, present and future. British Journal of Guidance and Counselling, Volume 28, No. 4, November 2000. 